\title{
Calidad de frutos en tomate (Solanum lycopersicum L. cv. Rocío) producidos bajo coberturas reflectiva y plástica
}

\author{
Fruit quality of tomato (Solanum lycopersicum L. cv. Rocío) \\ grown under reflective and plastic cover
}
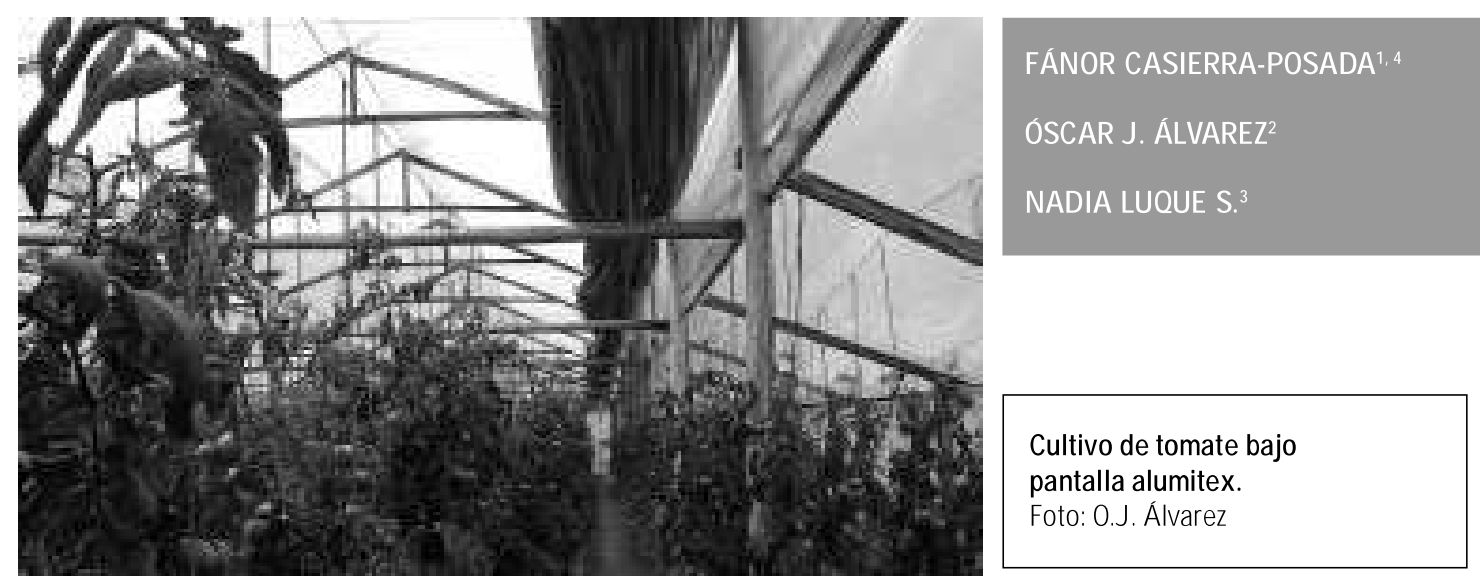

\section{RESUMEN}

Colombia produjo 476.985 t de tomate en el 2007; el departamento con mayor área sembrada fue Cundinamarca,

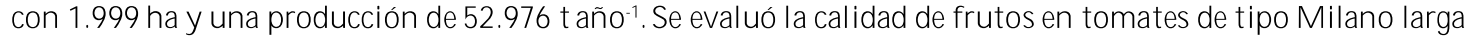
vida, cultivados en Susa, Colombia, a 2.655 msnm y sometidos a la influencia de las coberturas térmicas (tejido plateado reflectivo alumitex $50 \%$ o plástico transparente calibre seis). La cobertura alumitex aumentó la temperatura $2,01^{\circ} \mathrm{C}$ y disminuyó la humedad relativa 3,03\%, frente al testigo sin cubrimiento, y la cobertura plástica aumentó $0,83^{\circ} \mathrm{C}$ la temperatura promedio y redujo la $\mathrm{HR} 4,26 \%$, en comparación con su testigo. Los parámetros de calidad se vieron afectados positivamente con la utilización de las coberturas durante la noche. Los frutos fueron colectados y pesados en un estado de maduración $50 \%$ verdes-50\% rojos; cuando alcanzaron el color $100 \%$ rojo se les realizó pruebas para calcular firmeza del fruto, acidez total titulable, pH y contenido de sólidos solubles totales (SST); también fueron pesados nuevamente para determinar la pérdida de agua. LoS frutos influenciados por la cobertura aluminizada presentaron 29,3\% menor contenido de SST, fueron 0,86\% menos ácidos $(\mathrm{pH})$, perdieron 2,47\% menos humedad, fueron 5,01\% más firmes, aumentaron 30,66\% la acidez total titulable (ATT) y mostraron 51,62\% menor índice de madurez (SST/ATT). Con el uso del plástico, los frutos aumentaron 16,63\% el contenido de SST, fueron 1,22\% menos ácidos ( $\mathrm{pH}$ ), perdieron 8,94\% de humedad, aumentaron $4,53 \%$ la acidez titulable y $12,31 \%$ el índice de madurez, en comparación con los respectivos testigos.

Facultad de Ciencias Agropecuarias, Grupo de Investigación Ecofisiología Vegetal, Universidad Pedagógica y Tecnológica de Colombia, Tunja (Colombia).

2 Facultad de Ciencias Agropecuarias, Universidad Pedagógica y Tecnológica de Colombia, Tunja (Colombia).

3 Centro de Investigaciones y Asesorías Agroindustriales, Universidad Jorge Tadeo Lozano, Chía (Colombia).

4 Autor paracorrespondencia. fanor.casierra@uptc.edu.co 


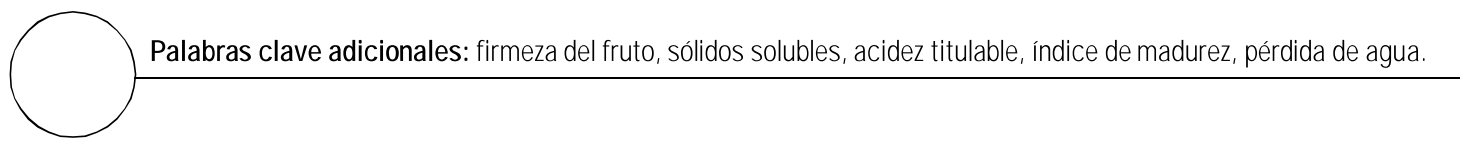

\section{ABSTRACT}

In Colombia, 476,985 t of tomato were produced in 2007. Cundinamarca was the principal region in terms of cultivated area, with 1,999 ha and 52,976 t year $^{-1}$. The quality of Milano tomatoes harvested in Susa, Colombia, at 2,655 $\mathrm{m}$ a.s.l., was evaluated. The fruits were influenced by thermal screens (reflective plastic tissue, Alumitex $50 \%$ and size 6 transduced plastic screen). The Alumitex screen increased the temperature by $2.01^{\circ} \mathrm{C}$, and decreased the relative humidity by $3.03 \%$, compared to the control plants without coverage. The plastic screen increased the temperature by $0.83^{\circ} \mathrm{C}$ and decreased the relative humidity by $4.26 \%$ compared to the control. Quality parameters were enhanced by coverage use during the night. The fruits were collected and the weight was estimated at the maturity stage $50 \%$ green-50\% red; subsequently when $100 \%$ red color was reached, the fruit firmness, total tritatable acidity (TTA), $\mathrm{pH}$ and total soluble solids (TSS) were measured. In addition, the fruit weight was measured again to determine water loss. The fruits affected by the aluminized screen showed a lower TSS (29.3\%), 0.86\% less acidity (pH), 2.47\% lower water loss, increased fruit firmness (5.01\%) and increased titratable acidity (30,66\%) and decreased maturity index 51,62\% (TSS/TAA). The fruits influenced by the plastic screen had increased TSS content (16.63\%), TTA (4.53\%) and maturity index (12.31\%), and reduced $\mathrm{pH}(1.22 \%)$ and water loss (8.94\%) as compared to the control fruits.

Additional key words: termic screen, fruit firmness, soluble solids, titratable acidity, maturiy index, water loss.<smiles>C1CCCCCCCCC1</smiles>

Originario de América tropical, el tomate (Solanum lycopersicum L.) es la hortaliza que más se produce en el mundo; según datos de la Organización de las Naciones Unidas para la Agricultura y la Alimentación, en el 2007 su producción alcanzó la cifra de 123'790.000 taño1 , y China fue el principal productor mundial, al promediar 30,57 millones de toneladas anuales (25\% del total mundial), seguida de Estados Unidos, con 11,37 millones de toneladas ( $9 \%$ del total mundial) y de Turquía, con cerca de 9,70 millones de toneladas ( $8 \%$ del total mundial) (FAO, 2007). Colombia produjo $476.985 \mathrm{t}^{\text {año-1 }}$ en 2007, siendo el departamento de Cundinamarca el primer productor, con 52.976 t año-1 y un área sembrada de 1999 ha (MADR, 2009).

Algunos de los factores más limitantes en la producción nacional son los problemas fitosanitarios y el desarrollo de los cultivos, que algunas veces no se realizan con base en las condiciones climáticas de los ecosistemas intervenidos para la producción, a pesar de que existen variedades mejoradas resistentes a plagas y enfermedades, como la variedad Rocío, cuya planta cuenta con vigor mediano a alto, entrenudos cortos y muy precoces, alto potencial de rendimiento, fruto de cal ibre grande, de $280 \mathrm{a}$ $300 \mathrm{~g}$, excelente color y firmeza destacada, de tipo larga vida, y con características de resistencia al virus del mosaico del tabaco, a Fusarium (Fusarium oxysporum) razas 1 y 2, a verticillium (Verticillium dahliale) y nemátodos (Jaramillo et al., 2007). Sin embargo, la variedad Rocío se cultiva bajo condiciones protegidas mediante el empleo de cubiertas plásticas, la demanda de insumos agrícolas para su cultivo es alta y los rendimientos (25 $\mathrm{t} \mathrm{ha}^{-1}$ ) están por debajo del promedio mundial (36 t ha-1) (FAS-USDA, 2003); por esta razón es indispensable evaluar alternativas como la utilización de coberturas 
térmicas para el trópico frío, buscando controlar la temperatura, la humedad relativa y la luz, para esos cultivos establecidos bajo cubiertas plásticas, puesto que son los más utilizados por los productores colombianos.

Las pantallas térmicas aluminizadas se extienden sobre un cultivo con el fin de producir un efecto térmico, cerrando el paso de energía calórica infrarroja y reflejándola sobre él; el aluminio funciona como una barrera infrarroja, caso contrario a las pantallas de polietilenos o policarbonados. De igual forma, si la pantalla es térmica se utiliza únicamente en la noche, este es el caso de la pantalla plástica, pero si la pantalla es reflectora, como la pantalla alumitex, se puede utilizar durante la noche, para evitar la pérdida de energía por radiación infrarroja, y en el día, para evitar el calentamiento del cultivo (Anglés, 2001; Szpiniak, 2002). Para buscar un efecto de reflexión de luz, la hebra de aluminio debe ser torneada, con el objeto de que una vez que la radiación solar incida sobre la malla, esta se caliente por la acción de los rayos infrarrojos, y, al mismo tiempo, parte de la luz se refleje, pero ya sin el calor; esta luz sin energía calórica incidirá como luz difusa en las hojas para aumentar la temperatura.

Las heladas se caracterizan por noches muy claras y extremadamente frías. En un día soleado, la planta absorbe la energía, y durante las noches claras reduce la radiación con respecto al frío del ambiente; al no haber obstáculos contra la onda larga, la planta pierde su calor y su temperatura desciende al nivel de la temperatura ambiente, incluso a menos de $0{ }^{\circ} \mathrm{C}$. Hay muchas plantas que mueren después de una noche de temperaturas muy frías (Anglés, 2001; Castilla, 2000; Szpiniak, 2002). La malla alumitex bloquea la radiación emitida por las plantas, reflejándola nuevamente sin energía calórica de vuelta hacia las plantas y el suelo. La temperatura de la hoja se mantiene sobre los $0{ }^{\circ} \mathrm{C}$, mientras la temperatura del aire puede Ilegar a ser $-6{ }^{\circ} \mathrm{C}$ (Anglés, 2001; Szpiniak, 2002).

Los factores que determinan el crecimiento vegetal son la temperatura, la actividad fot osintética, el porcentaje de humedad, el $\mathrm{CO}_{2}$ y la radiación; factores que se pueden optimizar con el uso de las pantallas térmicas, que además contribuyen al ahorro energético en los invernaderos con calefacción, principalmente donde existen estaciones. En verano, las pantallas se cierran durante el día, y en invierno se cierran en las noches, para mantener la temperatura y lograr un ahorro de combustibles de un $20 \%$ en los invernaderos con calefacción (Raposo-Lobet, 2004). El cultivo en invernaderos con calefacción exige, en invierno, un consumo medio anual de 20 a $45 \mathrm{~m}^{3}$ de gas natural o de 15 a $45 \mathrm{~kg}$ de gasóleo por metro cuadrado (Vilarnau, 1994).

Básicamente, la utilización de la pantalla depende directamente de la radiación y la temperatura. Si la radiación solar exterior supera la consigna de radiación, se extiende la pantalla térmica para sombrear; si desciende por debajo de la consigna menos el diferencial, se recoge. Por otro lado, si la radiación solar exterior es próxima a cero, la función de la pantalla térmica se realiza en función de la temperatura: si la temperatura del invierno desciende por debajo de la consigna de temperatura, se extiende la pantalla para disminuir las pérdidas de energía; si aumenta, se recoge (Oliveira et al., 2001).

La mayoría de trabajos sobre la utilización de coberturas aluminizadas se han adelantado en Almería, España. En una de estas investigaciones se evaluó la ventaja de utilizar las coberturas aluminizadas al 40,50 y $60 \%$, frente a un testigo con cobertura tradicional, en una densidad de 1,78 plantas $\mathrm{m}^{-2}$ y utilizando una cubierta de polietileno termoaislante de 200 $\mu \mathrm{m}$; se concluyó que los frutos de tomate más firmes, pero definitivamente con menor contenido de SST, fueron aquellos sometidos a sombrío durante las horas del día en los veranos con alta incidencia de radiación directa y cubiertos o protegidos en las noches con bajas temperaturas de invierno (Callejón-Ferre et al., 2009). De igual manera, el trabajo desarrollado por Kenig y Fernández-Rodríguez (2002), donde se evaluó este mismo material aluminizado con iguales porcentajes de sombrío, evidenció que los racimos de tomate cosechados bajo este sistema mejoran la calidad en general, siendo el mejor tratamiento aquel con $40 \%$ de sombrío; además, el número de frutos por racimo no se vio afectado por la cantidad de sombra proporcionada por la cobertura térmica. 
En la búsqueda de un mejor control en el manejo del clima, para optimizar recursos y aumentar la productividad durante el proceso de producción de tomate, en ocasiones se pueden afectar los atributos físicos y químicos de los frutos cosechados, principalmente si se intervienen factores como la luz, la radiación y la temperatura mediante el empleo de coberturas plásticas, pantallas térmicas y mallas en aluminio, generándose mermas o mejorías de la calidad organoléptica de los tomates. Por tanto, se hizo necesario realizar pruebas de calidad, tales como firmeza, forma, tamaño, madurez, $\mathrm{pH}$, contenido de SST y pérdida de humedad, a Ios frutos cosechados de plantas de tipo milano variedad Rocío, sometidas a la utilización de las coberturas térmicas durante la noche en el municipio de Susa-Cundinamarca, Colombia-.

\section{MATERIALES Y MÉTODOS}

La investigación se llevó a cabo en dos fincas, ambas situadas en zona rural del municipio de Susa: San Carlos (invernadero 1), donde se empleó la cobertura alumitex, y Las Palmas (invernadero 2), donde se evaluó la cobertura plástica. Dichos invernaderos se encontraban a una altura de 2.655 y 2.663 msnm, respectivamente, con una temperatura promedio de $15 \pm 3^{\circ} \mathrm{C}$ y una humedad relativa del $82 \pm 5 \%$; además, se contó con el laboratorio de la Gobernación de Boyacá, ubicado en la Universidad Pedagógica y Tecnológica de Colombia, el cual presenta una temperatura promedio de $15 \pm 2^{\circ} \mathrm{C}$ y una humedad relativa de $71 \pm 5 \%$ y está ubicado a $2.690 \mathrm{msnm}$. Para tener un registro más acertado, se llevaron datos de temperatura húmeda y seca a lo largo del ciclo del cultivo, y se utilizaron varios Cox Tracer (Permanet Memory Temperatura Recorder), registrando las temperaturas húmedas y secas dentro de las coberturas en cada sección con pantallas y sin pantallas; de igual manera, se llevó registro en la parte externa de las áreas cosechadas, y se hizo seguimiento a las calidades de los frutos cosechados, principalmente se registró la presencia de frutos malformados en las cosechas realizadas cada $8 \mathrm{~d}$.

Las dos coberturas fueron extendidas a $3 \mathrm{~m}$ sobre el suelo, formando un techo paralelo sobre un total de 2.000 plantas de tomate tipo milano variedad Rocío, en un área aproximada de 400 $\mathrm{m}^{2}$ para cada ensayo; cada una se extendió diariamente a las 5:00 p.m. y se recogió a las 7:00 a.m. Las coberturas presentaron las siguientes especificaciones técnicas:

La pantalla térmica alumitex. Es una tela alumitex de malla tejida en polietileno de alta densidad, recubierta con aluminio e incorporada con aditivos para mejorar su resistencia a condiciones ambientales; genera un $50 \%$ de sombrío, reduce la temperatura durante las horas diurnas, desplazando las temperaturas extremas (temperaturas máximas y mínimas), y permite la permeabilidad al agua, optimizando el agua de fertiriego y reduciendo el exceso de sales en determinados suelos; también aumenta el confort climático de las plantas, mejorando el proceso de fotosíntesis con mayor difusión de luz, permitiendo un sombrío uniforme. Esta pantalla se ubicó entre las plantas y la cobertura plástica.

La pantalla térmica plástica. Es un plástico agrolene verde calibre 6, que bloquea el 100\% de la radiación ultravioleta UV (200-340 nm) y presenta resistencia máxima de exposición al azufre de $4.000 \mathrm{mg} \mathrm{L}^{-1} \mathrm{ppm}$ y resistencia máxima de exposición al cloro de 150 ppm; tiene una transmisión de luz mínima de $90 \%$, con un efecto de difusión de luz máxima de $23 \%$, según la norma ASTM D-1003.

Se realizaron los correspondientes análisis de suelos para lograr una adecuada fertilización a lo largo del cultivo; se llevaron registros de temperatura húmeda y seca, para calcular la humedad relativa durante el ciclo del cultivo, y se hicieron constantes monitoreos fitosanitarios desde la siembra hasta la cosecha, para garantizar el adecuado desarrollo de las plantas de tomate y minimizar el impacto de plagas y enfermedades que pudieran afectar el normal desarrollo fisiológico del cultivo.

Se hicieron dos experimentos independientes: en el primero (invernadero 1) se evaluó la pantalla alumitex y el testigo, y en el segundo (invernadero 2), la pantalla plástica y el testigo: para ambos casos se utilizó un diseño completamente al azar, con un arreglo factorial 
constituido por la presencia o no de las coberturas térmicas durante cuatro cosechas, correspondiente a cuatro repeticiones del ensayo.

Este ensayo permitió evaluar la calidad fisicoquímica de 480 frutos colectados y pesados de forma individual, en un estado de madurez de cosecha, cuando los frutos presentaron $50 \%$ de color rojo y $50 \%$ de color verde, correspondiente a la forma tradicional de cosecha por los productores de la zona. Las lecturas de firmeza, ATT, pH y SST se realizaron cuando los frutos alcanzaron la madurez de consumo, es decir, cuando completaron totalmente el color rojo, después de pasar 16 días a partir de la cosecha a una temperatura ambiente en el laboratorio. La diferencia entre el peso al momento de la cosecha y el peso al momento de la madurez de consumo permitió calcular la pérdida de humedad durante el almacenamiento, determinando la mayor evapotranspiración de los frutos estudiados.

El ensayo para evaluar la calidad fisicoquímica correspondió a cuatro cosechas, cada una con un número total de 120 frutos distribuidos en los tratamientos con y sin pantalla (invernadero 1 y 2):

Tratamiento 1: 30 frutos colectados de plantas sometidas a la pantalla de alumitex (invernadero 1): tratamiento 2: 30 frutos colectados de plantas en la sección donde no había pantalla alumitex (invernadero 1); tratamiento 3: 30 frutos colectados de plantas sometidas a la pantalla de plástico (invernadero 2); tratamiento 4: 30 frutos colectados de plantas en la sección donde no hay pantalla plástica (invernadero 2).

De los 30 frutos colectados en cada uno de los tratamientos (pantallas térmicas y sus respectivos testigos), 10 correspondieron a un tamaño extra; 10, a un tamaño primera, y 10, a un tamaño de segunda, cosechando dos frutos de cada diámetro por planta a cinco plantas tomadas aleatoriamente durante las cuatro cosechas, para un total de 20 plantas por cada sección (tabla 1).

Los resultados fueron sometidos a un análisis clásico de varianza y posteriormente se realizó la prueba de comparación de promedios de Tukey $(P \leq 0,05)$, utilizando el programa SPSS ${ }^{\circledR}$ versión
Tabla 1. Categorías de clasificación de los frutos de acuerdo con su diámetro.

\begin{tabular}{|l|l|}
\hline \multicolumn{1}{|c|}{ Categoría } & \multicolumn{1}{c|}{ Diámetro } \\
\hline Extra & $>8 \mathrm{~cm}$ \\
\hline Primera & $>7 \mathrm{~cm}$, pero $<8 \mathrm{~cm}$ \\
\hline Segunda & $>6 \mathrm{~cm}$, pero $<7 \mathrm{~cm}$ \\
\hline
\end{tabular}

11.5.1 (Statistical Product and Service Solutions, Chicago, IL - Nov. 2002).

Las variables medidas fueron: peso $(\mathrm{g})$ con respecto al diámetro en el día de la cosecha, cantidad de sólidos solubles totales (SST o ${ }^{\circ} \mathrm{Brix}$ ), $\mathrm{pH}$ del jugo de los frutos, pérdida de humedad (\%), firmeza de la pulpa (Ib pulg-2), acidez total titulable y relación de madurez (SST/ATT). Los datos de firmeza del fruto se obtuvieron con un penetrómetro manual Bertuzzi FT 327 (3 27 L); se tomaron lecturas a 480 frutos en el diámetro ecuatorial; el contenido de SST se determinó con un refractómetro, y los resultados del contenido de acidez total titulable (ATT) se presentaron como porcentaje de ácido cítrico, de acuerdo con los protocolos mencionados por Gull et al. (1982).

\section{RESULTADOS Y DISCUSIÓN}

Durante el ensayo, la cobertura alumitex indujo un incremento de $2,01^{\circ} \mathrm{C}$ en la temperatura dentro del invernadero, mientras que redujo en $3,03 \%$ el contenido de vapor de agua. Por otra parte, la implementación de cobertura plástica aumentó en menor cantidad la temperatura $\left(0,83^{\circ} \mathrm{C}\right)$, y el contenido de humedad se redujo $4,26 \%$ (tabla 2).

Se menciona que la temperatura afecta la actividad metabólica celular, la absorción de agua y nutrientes, el intercambio gaseoso, la producción y gasto de carbohidratos y la biosíntesis de reguladores del crecimiento, entre otros (Tognoni, 2000). Un factor limitante en la producción de tomate en Susa corresponde a unas bajas temperaturas durante la noche; sin embargo, el uso de las coberturas no siempre es benéfico, porque se prolonga el ciclo del cultivo por efecto de sombrío y se aumenta la caída de flores, debido, principalmente, a que se ve 


\begin{tabular}{|l|c|c|c|c|}
\hline \multicolumn{5}{|c|}{ Tabla 2. Temperatura y humedad relativa promedio durante el ciclo cultivo en los invernaderos. } \\
\hline \multicolumn{1}{|c|}{ Tratamiento } & Con pant. alumitex & Sin pant. alumitex & Con pant. plástica & Sin pant. plástica \\
\hline Temperatura $\left({ }^{\circ} \mathrm{C}\right)$ & 16,52 & 14,51 & 16,12 & 15,29 \\
\hline Humedad relativa (\%) & 84,11 & 87,14 & 78,16 & 82,42 \\
\hline
\end{tabular}

afectada la tasa de asimilación fotosintética, alterándose de forma directa la asimilación de nutrientes hacia los vertederos (Gent-Martin, 2003); no obstante, en este caso no fue así; el aumento en la temperatura durante la noche tuvo un efecto positivo, porque se presentó un mayor cuajado de frutos con una mejor apariencia física (menos malformaciones), reflejado en un aumento en la cosecha de 13,3\% (pantalla alumitex) y $11,4 \%$ (pantalla plástica) en comparación con los respectivos testigos.

\section{Contenido de sólidos solubles totales}

Se pudo determinar un efecto significativo $(P \leq 0,05)$ con el uso de las coberturas sobre el contenido de SST en Ios frutos. El uso de la cobertura alumitex redujo en $29,3 \%$ el contenido de SST respecto al testigo. De forma contraria, se observó que el contenido de SST en los frutos bajo cobertura plástica aumentó $16,6 \%$ en comparación con aquellos sin efecto de la cobertura (figura 1).
En la presente investigación el contenido de sólidos solubles totales estuvo entre 2 y $3^{\circ}$ Brix, valores por debajo de los reportados por AguayoGiménez y Artés-Calero (2004), quienes encontraron valores para SST entre 4,5 y 5,5 ${ }^{\circ} \mathrm{Brix}$ y los consideraron óptimos en cuanto a calidad para los frutos de tomate. Posiblemente este comportamiento obedeció a la variedad de tipo milano larga vida (Rocío), material con el que se desarrolló la investigación, ya que sus propiedades organolépticas están enfocadas a un bajo contenido de azúcares, por tratarse de variedades de mesa; por otro lado, lecturas tomadas 16 días después de la cosecha tienden a ser más bajas, según Reina et al. (1998), quien explicó el comportamiento de los sólidos solubles totales a lo largo de la maduración del fruto, ya que a partir del octavo día después de la cosecha Ios SST disminuyen de forma considerable, porque los hidratos de carbono sufren cambios bioquímicos durante la maduración, y la degradación de los polisacáridos de las membranas celulares ejerce una contribución importante sobre un mayor contenido de

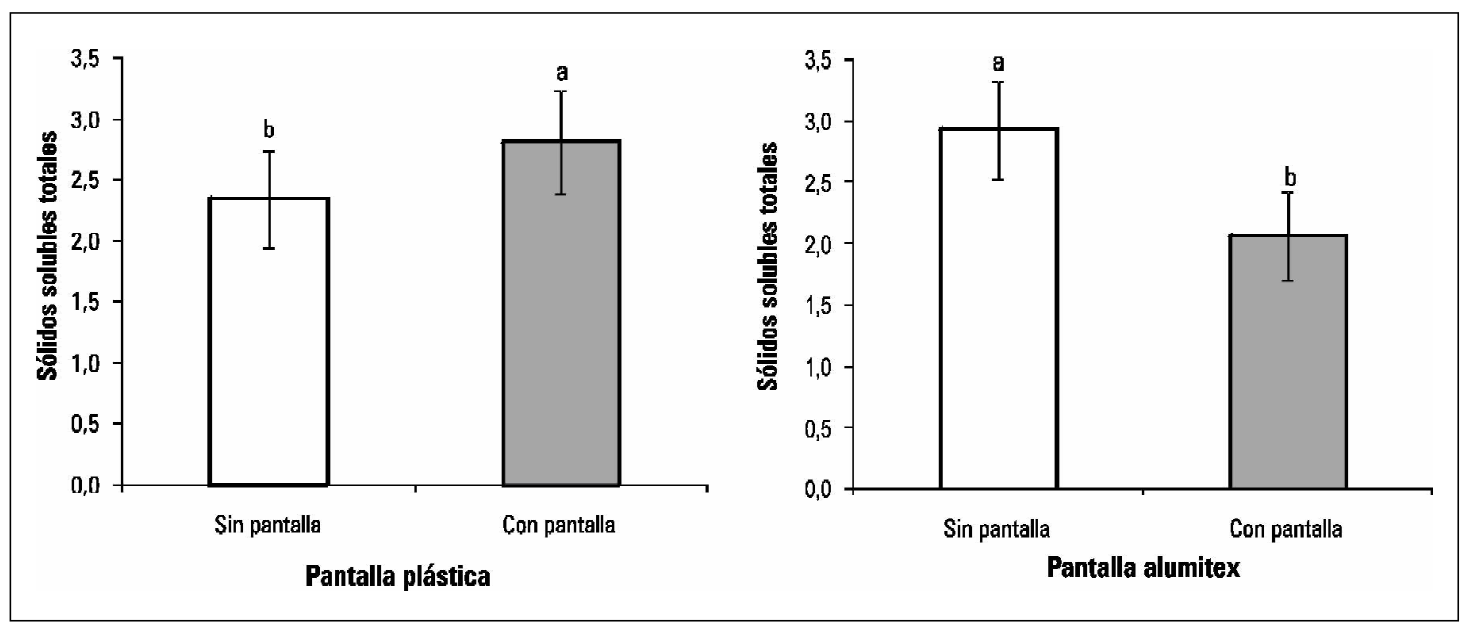

Figura 1. Contenido de sólidos solubles totales ( ${ }^{\circ}$ Brix) en frutos de tomate cosechados de plantas cultivadas bajo la influencia de las coberturas alumitex y plástica. Promedios con letras distintas indican diferencia significativa según la prueba de Tukey $(P \leq 0,05)$; de igual manera, se indica la desviación estándar por exceso y por defecto. 
azucares, pero a través del tiempo transcurrido Ios SST disminuyen. Kurahashi y Takahashi (1995) afirmaron que los frutos expuestos relativamente a una iluminación intensa tienden a ser más dulces, al presentar mayor contenido de SST, que los frutos que crecen a la sombra; puesto que la planta es más eficiente fotosintéticamente, también aumenta la evapotranspiración, permitiéndole intercambio gaseoso que mejora el metabolismo y la formación de azúcares. La cobertura aluminizada afectó las propiedades fisicoquímicas de los frutos pues fueron más firmes y presentaron un menor contenido de sólidos solubles totales, con referencia a los cosechados en el área sin cobertura. Resultados similares fueron reportados por Callejón-Ferre et al. (2009), donde el aumento en el contenido de SST se dio con la utilización de la cobertura aluminizada con un sombrío del $40 \%$, superando a las coberturas con valores de 50 y 60\%; a mayor efecto de sombra en las plantas, hay un menor contenido de grados Brix en los frutos de tomate.

En esta investigación, el bajo contenido de SST para los dos tipos de cobertura se pudo deber a que el registro de los datos se realizó cuando los frutos presentaron un color $100 \%$ rojo, entre los días 15 y 16 después de la cosecha; además, porque los tomates larga vida para mesa tienden a presentar menor contenido de SST.
Es determinante el efecto de las coberturas sobre el contenido de sólidos solubles totales; la cobertura plástica induce mayor contenido de SST, mientras que la cobertura alumitex tuvo como efecto frutos con menor contenido de SST.

\section{Firmeza de la pulpa}

Se presentaron diferencias significativas $(P \leq 0,05)$ en la firmeza de frutos cultivados bajo la influencia de la cobertura alumitex (figura 2); se encontró que los frutos cosechados bajo este tipo de cobertura fueron más firmes en un $5 \%$ con respecto a su testigo. La cobertura plástica no influyó en la firmeza de los frutos.

Callejón-Ferre et al. (2009) y Kenig y FernándezRodríguez (2002) utilizaron coberturas aluminizadas con diferente porcentaje de sombrío (40, 50 y 60\%) en las horas de la noche durante el invierno y el otoño, para evitar las pérdidas de radiación infrarroja de onda larga, y en el verano durante el día, para reducir la radiación; los resultados indicaron que los frutos cosechados de plantas expuestas a un sombrío del $60 \%$ presentaron diferencias altamente significativas en cuanto a firmeza y contenido de sólidos solubles totales. Los tomates más firmes, pero con menor contenido de sólidos solubles totales, fueron aquellos expuestos a

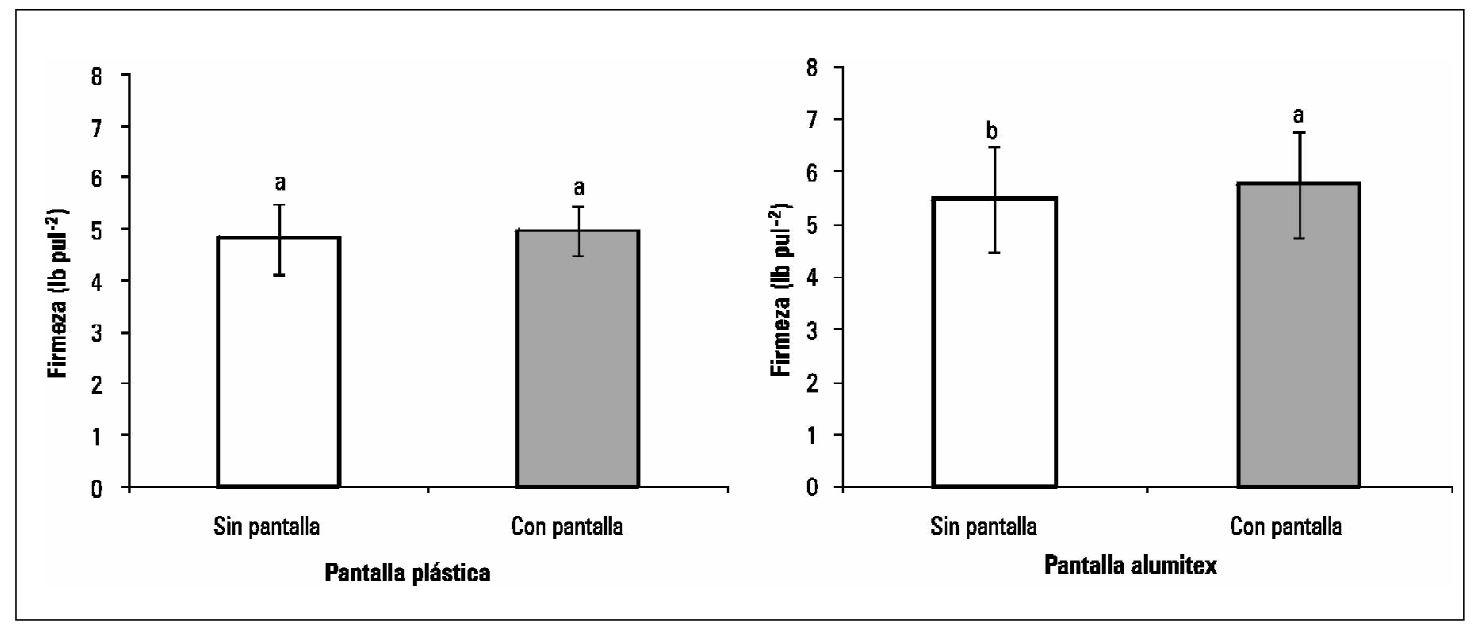

Figura 2. Firmeza de la pulpa de frutos cosechados de plantas cultivadas bajo la influencia de las coberturas plástica y alumitex. Promedios con letras distintas indican diferencia significativa según la prueba de Tukey $(P \leq 0,05)$; de igual manera, se indica la desviación estándar por exceso y por defecto. 
mayor porcentaje de sombra. En un trabajo realizado en manzano (Malus domestica Bork.), se evaluó el efecto del sombrío utilizando dos tipos de malla: 15 y 55\% de reducción de luz, y se demostró que no hubo diferencias significativas en cuanto a la firmeza, sin embargo, se aclara que los frutos colectados en las partes inferiores de las plantas, donde la luz fue menos directa, fueron más firmes (Dussi et al., 2005).

En el presente ensayo, los frutos expuestos a condiciones de sombrío manifestaron un retraso en el proceso de maduración, caso contrario a la cobertura plástica, con la cual las plantas reciben cantidades normales de rojo lejano que pueden ser aprovechadas por la planta para su fotosíntesis. Es importante destacar que la pérdida de firmeza es resultante de la acción de tres enzimas principalmente: celulasa, pectinesterasa y poligalacturonasa (Tucker et al., 1980), siendo esta última la que mejor se correlaciona con el ablandamiento. Ramírez et al. (2004) estudiaron los efectos de diferentes temperaturas sobre el contenido de etileno, PG (poligalacturonasa) y ACC (ácido-1aminocilopropano-1-carboxílico). Los frutos almacenados a temperaturas inferiores al ambiente mostraron un mayor peso y firmeza; de igual manera, la producción de etileno, ACC y PG fue menor, alargando la vida en anaquel; por consiguiente, los frutos cosechados bajo la influencia de la cobertura aluminizada, por ser más firmes y perder menor contenido de agua en poscosecha, presentan posiblemente una menor producción de $A C C$, etileno y $P G$, disminuyendo la velocidad en el proceso de maduración y prolongando la vida útil del fruto. La cobertura plástica no afectó la firmeza de los frutos. Adicionalmente, los frutos cosechados bajo este tipo de material plástico perdieron menos agua durante la investigación, mejorando la calidad y la vida útil en poscosecha.

\section{pH del jugo}

En cuanto al $\mathrm{pH}$, se encontraron diferencias altamente significativas $(P \leq 0,05)$ con la utilización de cualquiera de las dos coberturas: los frutos cosechados con las coberturas tuvieron tendencia a ser ménos ácidos (figura 3): 0,86\% con la cobertura alumitex y $1,22 \%$ con la cobertura plástica.

Después de la cosecha, el pH en frutos de tomate reporta severas disminuciones, desde cuando los frutos son verdes o inmaduros hasta un estado de madurez fisiológica; luego, el $\mathrm{pH}$ se incrementa hasta alcanzar un estado de madurez de 100\% rojos, tanto en híbridos como en otras especies de Lycopersicon (Young et al., 1993). Los valores encontrados del pH hacen que los tomates estén

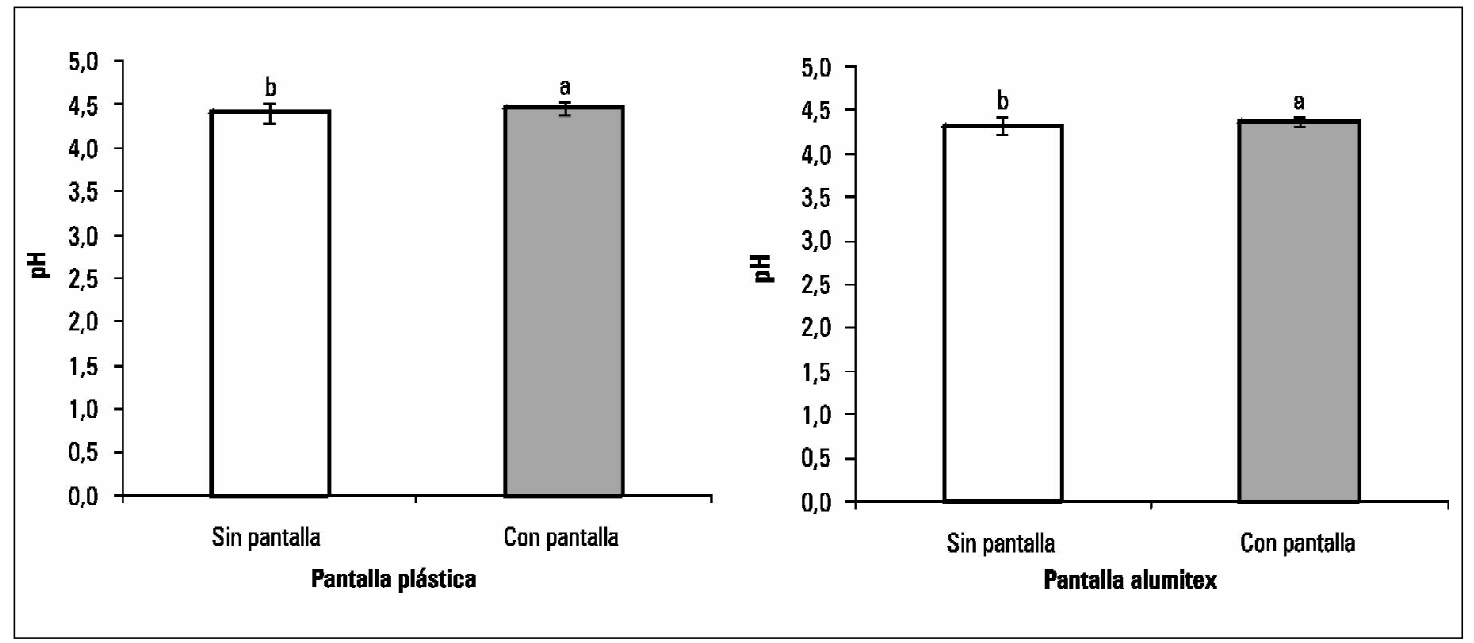

Figura 3. pH de frutos cosechados de plantas cultivadas bajo la influencia de las coberturas plástica y alumitex. Promedios con letras distintas indican diferencia significativa según la prueba de Tukey $(P \leq 0,05)$; de igual manera, se indica la desviación estándar por exceso y por defecto. 
dentro del rango de buena calidad (AguayoGiménez y Artés-Calero, 2004); posiblemente los frutos que tienden a ser ácidos tienen la posibilidad de ser menos susceptibles al ataque de patógenos (Ciruelos-Calvo et al., 2008).

El comportamiento del pH reportado por Keing y Fernandez-Rodríguez (2002) coincide con lo encontrado en este trabajo; el pH más alto se observó en los frutos sometidos al mayor porcentaje de sombrío, y fue mayor la reducción de la acidez en los frutos bajo cobertura plástica. Hay que aclarar que existen varios factores que afectan el pH de los frutos; uno de ellos son los niveles óptimos de fertilización de potasio de 220 $\mathrm{kg} \mathrm{ha}^{-1}$ (Ruiz et al., 2008). Al tomar el valor de $\mathrm{pH}$ del jugo de los frutos y observar la tendencia a ser más básicos, se asume que hay mayor translocación de bases como calcio y potasio a Ios vertederos, en este caso los frutos; sin embargo, a pesar de que existe un efecto de sombrío leve en las primeras horas de la mañana y al finalizar la tarde, el incremento en la temperatura fue determinante para la traslocación de los nutrientes.

\section{Acidez total titulable}

El contenido de ácidos orgánicos estuvo afectado significativamente $(P \leq 0,05)$ por la influencia del tipo de cobertura. El mayor contenido de ATT se observó en los frutos con cobertura alumitex, el cual fue $30,6 \%$ más alto que en los frutos sin cobertura aluminizada. De otra manera, Ios frutos cosechados bajo el efecto de la cobertura plástica incrementaron un 4,53\% el contenido de ácidos orgánicos respecto a las plantas control (figura 4).

Los ácidos orgánicos presentes en los alimentos influyen en su sabor, color y estabilidad. Los valores de acidez pueden ser muy variables en el tomate; el contenido de ácidos orgánicos aumenta con el crecimiento del fruto y se acumula principalmente en los lóculos; los principales ácidos son el málico y el cítrico, que representan alrededor del $13 \%$ de la materia seca. Al principio del crecimiento, el ácido málico es el predominante, mientras el cítrico representa sólo el 25\% (Nuez, 1995). Como en este trabajo se trata de frutos en estado de madurez de consumo, se asume que el contenido de ácido cítrico es mayor; por esta razón, la acidez titulable se expresó con relación a este.

Estudios preliminares han reportado la influencia de diferentes condiciones climáticas, como la iluminación y la temperatura, sobre la formación de ácidos orgánicos. Hamner et al. (1945) encontraron que plantas de tomate «Bonny Best», cultivadas en cinco localidades con condiciones agroecológicas distintas en los Estados Unidos, produjeron frutos con diferencias marcadas en el contenido de ácido ascórbico. Estas variaciones asociadas a la localidad no pudieron ser

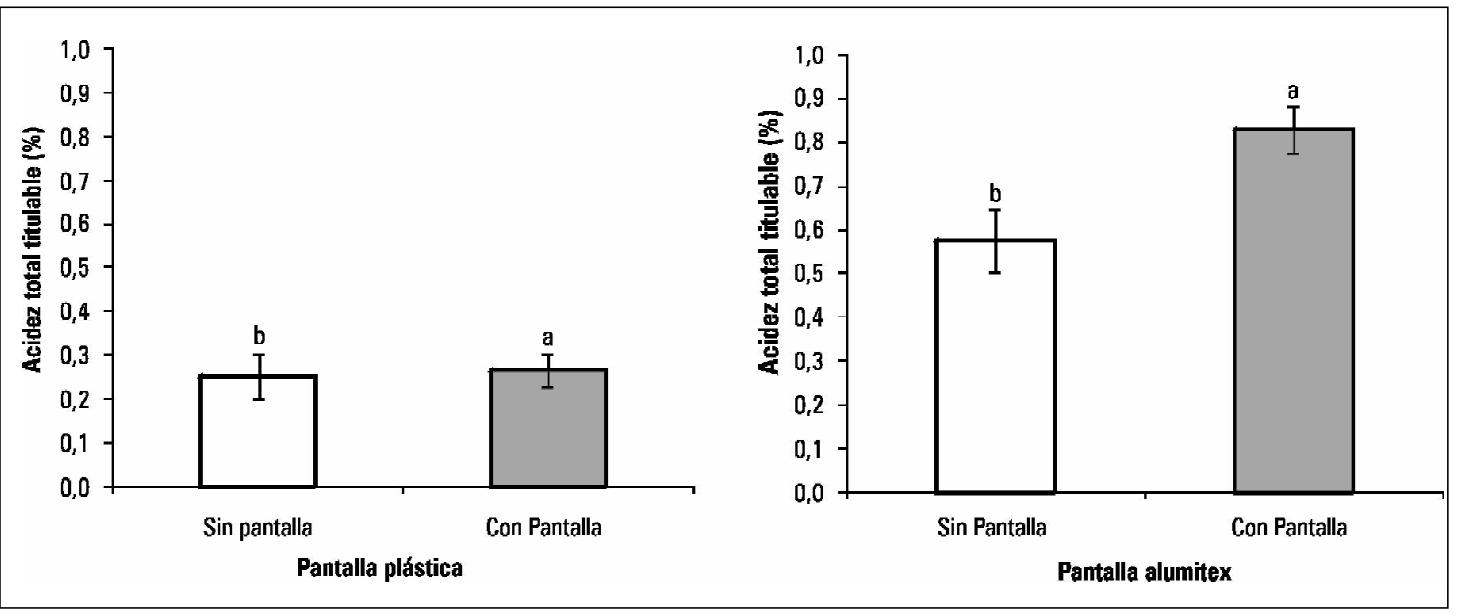

Figura 4. Acidez total titulable (\% de ácido cítrico) de frutos cosechados de plantas cultivadas bajo la influencia de las coberturas plástica y alumitex. Promedios con letras distintas indican diferencia significativa según la prueba de Tukey $(P \leq 0,05)$; de igual manera, se indica la desviación estándar por exceso y por defecto. 
correlacionadas con las condiciones del suelo o con las prácticas culturales, y presumiblemente se asociaron con la oferta ambiental. De hecho, en este trabajo se encontró que el porcentaje de sombrío en horas tempranas y la temperatura influyeron de forma positiva sobre la ATT, para los dos tipos de cobertura, a pesar de que fueron muy diferentes para las dos fincas.

Algunos autores han reportado que la calidad de luz es un factor determinante sobre el contenido de ácidos orgánicos en varios cultivos (Somers y Beeson, 1948). Hallsworth y Lewis (1944) estudiaron la relación existente entre la exposición de frutos de tomate al sol y su contenido de ácido ascórbico y determinaron que la cantidad de iluminación recibida directamente por los frutos influye sobre este ácido. Así como se había analizado en variables anteriores, existió un efecto de sombrío por parte de las pantallas sobre las planta; por tal razón, es posible que el contenido de ácidos orgánicos en los frutos haya sido afectado por el sombrío. Somers y Beeson (1948) indicaron que la cantidad de sombrío afecta significativamente la producción de ácidos orgánicos, más aún, que el contenido de acidez está determinado por el órgano de la planta que estuvo sombreado; es así como la iluminación directa a los frutos de tomate induce una producción alta de ácido ascórbico, y es mayor el contenido de ácidos orgánicos cuando las plantas reciben sombra y luz de manera alterna. Los frutos cosechados de plantas bajo la influencia de las coberturas alumitex y plástica presentaron un mayor porcentaje de ATT, porque las plantas recibieron luz durante el día y sombra en las primeras horas de la mañana, sin embargo, el valor de la acidez titulable estuvo dentro de los rangos aceptables (Djedidi et al., 1997); Ios contenidos de ácidos orgánicos disminuyen durante el proceso de maduración (Reina et al., 1998), indicando que los frutos cosechados bajo la influencia de la cobertura alumitex tendieron a madurar más despacio.

Se reporta que el comportamiento del $\mathrm{pH}$ a lo largo de la maduración del fruto es inversamente proporcional al contenido de acidez total titulable (Ruiz et al., 2008); sin embargo, para el presente ensayo, a pesar de que los frutos cosechados bajo la influencia de las coberturas fueron menos ácidos, también presentaron mayor contenido de ATT.

\section{Relación de madurez}

El incremento en sólidos solubles o la reducción de la acidez titulable provoca aumento en este indice de madurez, por tanto, crea un acercamiento a la madurez de consumo; esta es una forma de establecer el momento de la recolección. El índice de madurez está relacionado directamente con la dulzura y el sabor de los frutos. En este trabajo se presentaron diferencias altamente significativas en los dos tipos de cobertura $(P \leq 0,05)$; los frutos con menos dulce fueron los cosechados bajo la cobertura alumitex, mientras que los cosechados bajo la influencia de la cobertura plástica presentaron mayor dulzor. Se demostró que los frutos colectados con influencia de la cobertura plástica presentaron $12,31 \%$ mayor relación de madurez que el testigo, lo cual les confiere mejores propiedades organolépticas para el consumidor; a diferencia de la cobertura alumitex, donde los frutos fueron menos maduros al paladar, con un índice de cosecha 51,62\% menor, en relación con el testigo (figura 5).

Ya se había mencionado el efecto del sombrío sobre la firmeza, el contenido de sólidos solubles totales y el contenido de ácido cítrico; este efecto se vio reflejado en el índice de madurez calculado, donde los frutos fueron más dulces al cosecharse de plantas sometidas a la cobertura plástica, en comparación con el testigo. En cuanto a la cobertura alumitex, el bajo indicador de madurez posiblemente estuvo asociado de forma directa al momento en que se recogía la pantalla dentro del invernadero (7:00 a.m.).

Teniendo en cuenta que el tomate es un fruto climatérico (Rick, 1978), Casierra-Posada y Aguilar-Avendaño (2008) encontraron que el porcentaje de acidez titulable está relacionado con el híbrido y con el estado de maduración, porque los frutos cosechados muy temprano Ilevan a cabo plenamente los procesos bioquímicos que conducen a la transformación de los ácidos orgánicos en compuestos que determinan el sabor, reflejándose así en el índice de madurez, que aumentó cuando los frutos fueron cosechados en estados más tardíos. El comportamiento del índice de madurez a lo largo de la maduración fue muy similar cuando 


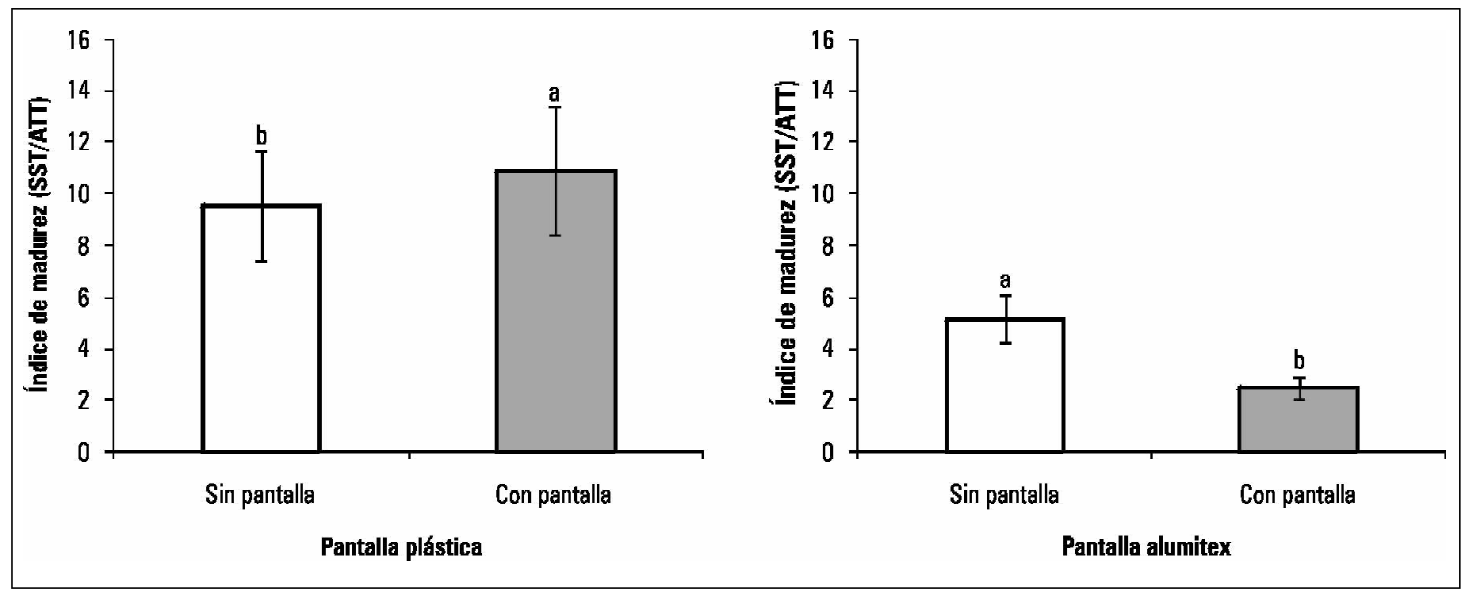

Figura 5. Índice de madurez de frutos cosechados de plantas cultivadas bajo la influencia de las coberturas plástica y alumitex. Promedios con letras distintas indican diferencia significativa según la prueba de Tukey $(P \leq 0,05)$; de igual manera, se indica la desviación estándar por exceso y por defecto.

se cosecharon $25 \%$ verdes- $75 \%$ rojos, $50 \%$ rojos-50\% verdes y $25 \%$ rojos- $75 \%$ verdes; por esta razón es acertado cosechar frutos cuando estén en un estado de coloración de $50 \%$ rojo y $50 \%$ verde. En plantas sometidas a la cobertura plástica, el efecto de la pantalla alumitex en las primeras horas de la mañana puede utilizarse como herramienta para demorar el proceso de maduración en épocas en las que hay bastante oferta de producto en el mercado, además también mejora la firmeza de los frutos.

\section{Pérdida de humedad}

La diferencia de las lecturas registradas bajo pantalla plástica y alumitex está relacionada con la transpiración del fruto, específicamente con la pérdida de humedad; el ensayo mostró diferencias altamente significativas $(P \leq 0,05)$ (figura 6).

Al aumentar la temperatura y al disminuir la humedad relativa, los frutos deben evapotraspirar en mayor cantidad. La pérdida de agua por

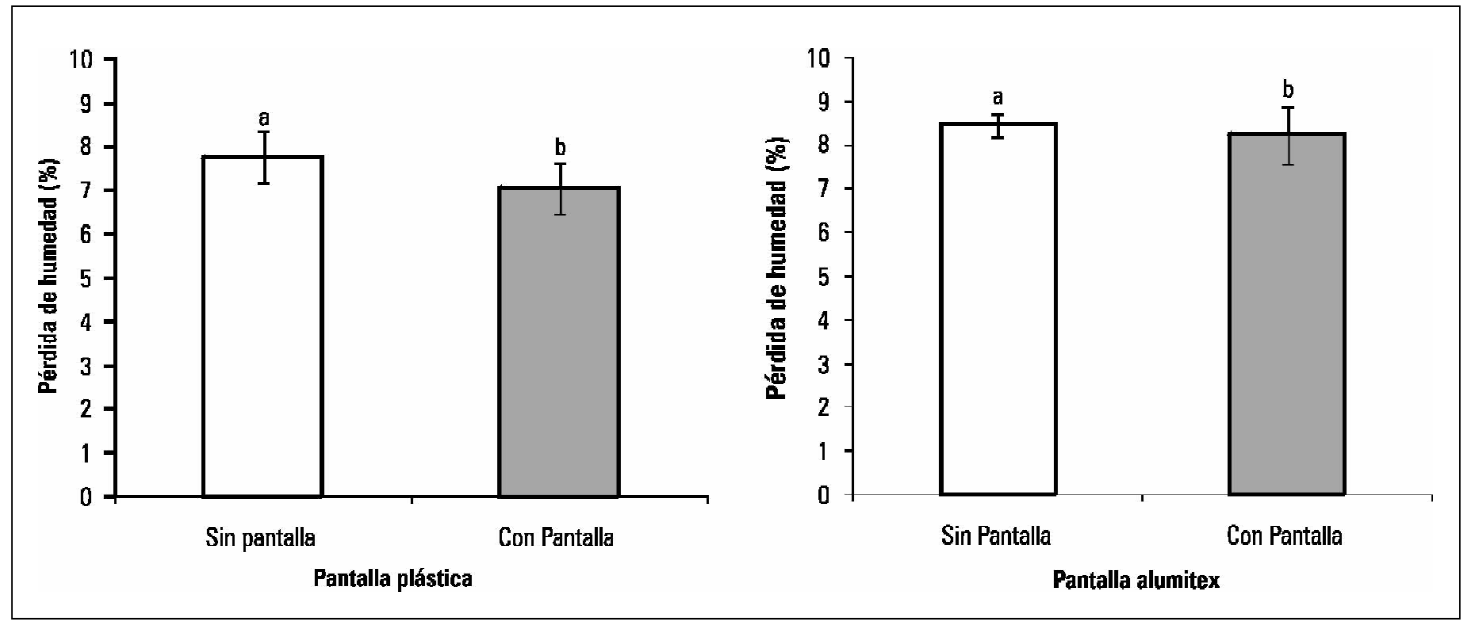

Figura 6. Pérdida de humedad de los frutos cosechados de plantas cultivadas bajo la influencia de las coberturas plástica y alumitex. Promedios con letras distintas indican diferencia significativa según la prueba de Tukey $(P \leq 0,05)$; de igual manera, se indica la desviación estándar por exceso y por defecto. 
transpiración fue menor en los sitios donde están ubicadas las coberturas alumitex y plástica, en un $2,47 \%$ y un $8,94 \%$, respectivamente. De cierta forma, la cobertura plástica hace que se mantenga un menor contenido de agua en vapor por $\mathrm{m}^{3}$ de aire, puesto que al aumentar la temperatura la humedad relativa disminuyó, ya que son inversamente proporcionales en los primeros días después de la cosecha. Al perder menor contenido de agua, los frutos cosechados bajo los dos tipos de coberturas presentan una mayor durabilidad después de colectados.

Los frutos cosechados bajo los dos tipos de cobertura perdieron menor contenido de agua, por lo cual alargaron su vida útil. Sin embargo, otros factores que pueden influir en la pérdida de humedad durante el almacenamiento de los tomates pueden ser el tamaño del fruto, daños en la corteza, humedad relativa, temperatura y movimiento del aire o ventilación (Pantastico, 1979).

\section{CONCLUSIONES}

El uso de la cobertura aluminizada redujo el contenido de sólidos solubles totales de los frutos, y el efecto de sombrío producido por la cobertura alumitex en las primeras horas de la mañana indujo frutos más firmes. Por otro lado, el uso de la cobertura plástica aumentó el contenido de azúcares totales en los frutos, ya que hubo una mayor degradación de polisacáridos reflejados en la acentuación de Ios SST; con la cobertura plástica, la firmeza de los frutos no se vio afectada.

Aunque la implementación de los dos tipos de cobertura incrementó significativamente el valor de $\mathrm{pH}$ en los frutos, este valor se mantuvo dentro del rango de lo normal, por tanto, no se consideró afectado este indicativo de calidad.

El contenido de ácidos orgánicos en los frutos se incrementó significativamente con el uso de las coberturas térmicas, producto de un retraso en el proceso de maduración de los frutos. La longevidad de los frutos cosechados fue mayor $y$, por consiguiente, es positivo el uso de las coberturas aluminizada y plástica.

Se determinó un ligero retraso en el proceso de maduración de los frutos cultivados bajo el efecto de la pantalla alumitex. Por otro lado, los frutos cosechados bajo la pantalla plástica presentaron mejores características organolépticas.

Los frutos que aumentaron la longevidad después de cosechados fueron los recolectados de plantas bajo el estímulo y el efecto de las coberturas térmicas.

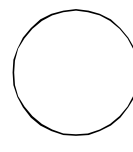

AGRADECIMIENTOS

Este trabajo hizo parte del proyecto Ilamado «Desarrollo de alternativas de manejo integrado del cultivo del tomate en sistemas de producción bajo invernadero en los municipios de Chía y Susa, en Cundinamarca, y Piedecuesta, en Santander», financiado por el Ministerio de Agricultura y Desarrollo Rural y el Fondo Nacional Hortofrutícola, Bogotá, adelantado por el Centro de Investigaciones y Asesorías Agroindustriales de la Universidad Jorge Tadeo Lozano, Chía. De igual manera fue indispensable el apoyo del Grupo de Investigación Ecofisiología Vegetal, de la Universidad Pedagógica y Tecnológica de Colombia, Tunja. 


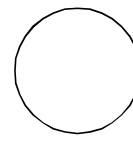

Anglés, M. 2001. Control climático ciclo de cultivo. Horticultura 152, 28-34.

Aguayo-Giménez, E. y F. Artés-Calero. 2004. Elaboración del tomate mínimamente procesado en fresco. Ediciones de Horticultura S.L., Reus, España.

Oliveira, C. E.L.; L. Luna; M.P. Muñoz-Cobo; J.L. García y R.M. Benavente. 2001. Calefacción y ahorro energético en invernaderos. Horticultura Global: Revista de Industria, Distribución y Socioeconomía Hortícola 151, 18-26.

Callejón-Ferre, Á.J.; F. Manzano-Agugliaro; M. DíazPérez; A. Carreño-Ortega y A. J. Pérez. 2009. Effect of shading with aluminized screens on fruit production and quality in tomato (Solanum lycopersicum L.) under greenhouse conditions. En: J. Agr. Res. 7(1), www.inia.es/ sjar; consulta: mayo de 2010

Casierra-Posada, F. y O.E. Aguilar-Avendaño. 2008. Calidad de frutos de tomate (Solanum lycopersicum L.) cosechados en diferentes estados de madurez. Agron. Colomb. 26(2), 298-305.

Castilla, F. 2000. Pantallas aluminizadas en el control climático de invernaderos. Horticultura 147 , $55-57$

Ciruelos-Calvo, A.; R. de la Torre y C. González-Ramos. 2008. Parámetros de calidad en el tomate para industria. pp. 149-169. En: La agricultura y la ganadería extremeñas, http://eia.unex.es/ $\mathrm{E}$ । I A A / P o r t a l s / 0 / La \% 20 Agricultura $\% 20$ y $\% 201$ a $\% 20$ Ganader\%C3\%ADa\%202007.pdf; consulta: 4 de abril de 2010

Djedidi, M.; D. Grasopoulos y E. Maloupa. 1997. The effect of different substrates on the quality of f Carmello tomatoes (Lycopersicon escullentum MILL) grown under protection in a hydroponic system. Chaiers Options Méditeranéenes 31, 379-83

Dussi, M. C.; G. Giardina y P. Reeb. 2005. Shade nets effect on canopy light distribution and quality of fruit and spur leaf on apple cv. Fuji. Span. J. Agric. Res. 3(2), 253-260.

FAO. 2007. FAOSTAT. Organización de las Naciones Unidas para la Agricultura y la Alimentación, División Estadística, Roma.

\section{REFERENCIAS BIBLIOGRÁFICAS}

Foreign Agricultural Service Department of Agriculture (FAS-USDA). 2003. Tomatoes \& Tomato Products. USDA: Horticultural \& Tropical products division. En: http:// w w w. f a s. us d a. gov/h t p / 2007\%20Tomato\%20article_08-24-07.pdf; consulta: 22 de mayo de 2010.

Gent-Martin, P.N. 2003. Effect of shade on quality of greenhouse tomato. Dept of Forestry \& Horticulture. En: http:// WWW.newenglandvfc.org/2003_conference/ proce ed ing s_ $03 /$ to mato/ effect_shade_quality_greenhouse_tomato.pdf; consulta: 13 de mayo de 2010

Gull, D.; Á. Cartagena y E. French. 1982. Análisis de calidad de tomate para lograr un mejor producto. Instituto Bolivariano de Tecnología Agropecuaria (IBTA Bolivia) y UFLA, La Paz.

Hallsworth, E.G. y V.M. Lewis. 1944. Some factors affecting the ascorbic-acid content of tomatoes in New South Wales. Empire J. Exp. Agr. 15, 132-147.

Hamner, K.; L. Bernstein y A. Maynard. 1945. Effects of light intensity, day length, temperature, and other environmental factors on the ascorbic acid content of tomatoes. J. Nutr. 29, 85-97.

Jaramillo, J.; V.P. Rodríguez; M. Guzmán; M. Zapata y T. Rengifo. 2007. Buenas prácticas agrícolas BPA en la producción de tomate bajo condiciones protegidas. Corpoica, FAO, Bogotá

Kenig, A. y E. Fernández-Rodríguez. 2002. Quality of truss tomatoes grown under aluminized reflective screens in Southern Spain. En: http:/ / w w w. polys a c k. com / files / 1f35d061c8702f3ebcdbc69136468942.pdf; consulta: 15 de mayo de 2010.

Kurahashi, T. y K. Takahashi. 1995. Comparison in light condition, fruit quality and photosynthetic rate between canopies of 'Fuji' apple trees trained to a Y-trellis and a central leader. J. Jap. Soc. Hort. Sci. 64(3), 499-508.

Ministerio de Agricultura y Desarrollo Rural (MADR) 2009. Análisis y estadísticas. Bogotá.

Nuez, F. 1995. El cultivo de tomate. Ediciones MundiPrensa, Madrid. 
Pantastico, E.B. 1979. Fisiología de la posrecolección, manejo y utilización de frutas y hortalizas tropicales y subtropicales. Ed. CECSA, México DF.

Ramírez, H.; L. I. Encina-Rodríguez; A. BenavidesMendoza; V. Robledo-Torres; J. HernándezDávila y S. Alonso-Corona. 2004. Influencia de la temperatura sobre procesos fisiológicos en postcosecha de tomate (Lycopersicon esculentum Mill.). Revista Agraria - Nueva Época 1(3), 3137.

Raposo-Lobet, C. 2004. Evaluación experimental y modelización del control de la pantalla térmica en invernaderos. Tesis de doctorado. Departamento de Ingeniería Rural, Escuela Técnica Superior de Ingenieros Agrónomos, Madrid.

Reina, C.E.; J.C. Guzmán-Torres y J.M. Sánchez . 1998. Manejo Poscosecha y evaluación de la calidad de tomate que se comercializa en la ciudad de Neiva. Universidad Surcolombiana, Neiva, Colombia.

Rick, C. 1978. The tomato. Sci. Amer. 239(2), 77-81.

Ruiz, S.C.A. 2008. Efecto del fertilizante potásico sobre la calidad química de frutos de tomate (Lycopersicon esculentun Mill.) almacenados bajo dos temperaturas. Revista Facultad de Agronomía 25, 286-302.
Somers, G F. y K.C. Beeson. 1948. The influence of climate and fertilizer practices upon the vitamin and mineral content of vegetables. Adv. Food Res. 1, 291-324.

Szpiniak, M. 2002. Pantallas termorreflectoras aluminizadas. En: http://www.polysack.com/ files/4ca736697b433150b0a83caf295cd4eb.pdf; consulta: 15 de agosto de 2010.

Tognoni, F. 2000. Temperatura. pp. 12-27.En: Memoria del Curso Internacional de Ingeniería, Manejo y Operación de Invernaderos para la Producción Intensiva de Hortalizas. Instituto Nacional de Capacitación para la Productividad Agrícola (INCAPA, S.C.), Guadalajara, México.

Tucker, G.A.; N.G. Robertson y D. Grierson. 1980 Changes in poligalacturonase isoenzimes during the ripening of normal and mutant tomato fruit. Biochem. 112,119-124.

Vilarnau, A. 1994. Energía sí, pero más limpia Horticultura Global. Revista de Industria Distribución y Socioeconomía Hortícola 99, 23 26

Young, T.E.; J. A. Juvik y J.G. Sullivan. 1993. Accumulation of the components of total solids in ripening fruits of tomato. J. Amer. Soc. Hort. Sci. 118, 286-292. 